\title{
Optical content and resolution of near-field optical images: Influence of the operating mode
}

\author{
R. Carminati, ${ }^{\text {a) }}$ A. Madrazo, and M. Nieto-Vesperinas \\ Instituto de Ciencia de Materiales, Consejo Superior de Investigaciones Científicas, Cantoblanco, \\ 28049 Madrid, Spain \\ J.-J. Greffet \\ Laboratoire d'Energétique Moléculaire et Macroscopique, Combustion, Ecole Centrale Paris, \\ Centre National de la Recherche Scientifique, 92295 Châtenay-Malabry Cedex, France
}

(Received 2 January 1997; accepted for publication 1 April 1997)

\begin{abstract}
Recent experimental work has shown that the contrast of near-field optical images depends on the path followed by the tip during the scan. This artifact may misguide the interpretation of the images and the estimation of the optical resolution. We provide a rigorous theoretical study of this effect based on three-dimensional perturbation theory and two-dimensional exact numerical calculations. We quantitatively study the dependence of the artifact on the illumination/detection conditions and on the scattering potential of the sample. This study should provide guidelines for future experimental work. (ㅇ 1997 American Institute of Physics. [S0021-8979(97)06813-8]
\end{abstract}

\section{INTRODUCTION}

Optical resolution beyond the Rayleigh limit has been demonstrated in the past ten years in scanning near-field optical microscopy (SNOM). ${ }^{1,2}$ Among the various techniques that have been proposed, two categories can be distinguished: illumination-mode and collection-mode SNOM. In illumination-mode SNOM, a tip (nanosource) locally illuminates the sample and one collects the field scattered into the far zone. ${ }^{3,4}$ Examples of nanosources are a tapered metalcoated optical fiber with an aperture at the tip apex, ${ }^{4}$ or the tetrahedral tip introduced recently. ${ }^{5}$ In collection-mode SNOM, the sample is illuminated by an extended field, as in classical microscopy, and the scattered near field is collected by a local probe. This probe can be the tip of an optical fiber, ${ }^{6}$ or a scattering tip as that used in apertureless SNOM. ${ }^{7,8}$ A particular case of collection-mode SNOM is the photon scanning tunneling microscope (PSTM or STOM) in which the sample is illuminated by an evanescent wave produced by total internal reflection. ${ }^{9}$

Let $\mathscr{S}(x, y, z)$ be the optical signal that is detected when the tip is located at the point $(x, y, z)$. The $z$ direction is chosen to be normal to the mean plane of the sample surface. In order to get sub-wavelength resolution, part of the signal must come from the conversion of evanescent waves into propagating waves. This holds whatever the technique. Thus, the tip (either illuminating or detecting) has to be kept at subwavelength distance from the sample during the scan. Three different operating modes have been used so far to regulate the tip-sample distance. (1) In the constant-height mode, the tip is moved in a plane $z=z_{0}$, and one records $\mathscr{S}\left(x, y, z_{0}\right)$. (2) In the constant-intensity mode, the optical signal $\mathscr{S}$ is kept constant with a feedback system, forcing the tip to follow a surface $z=h(x, y)$. This surface does not in general reproduce the topography of the sample. Recording the motion of the tip [i.e., the surface $z=h(x, y)$ ] produces the image. This mode has been used extensively in

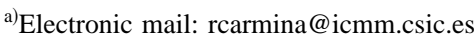

PSTM/STOM. ${ }^{9}$ (3) In the constant-distance mode, the tip is forced to follow a surface $z=f(x, y)$ by an auxiliary nonoptical distance-control mechanism. The optical signal that is recorded is $\mathscr{A}[x, y, f(x, y)]$. The distance-control mechanism can use a scanning tunneling microscope (STM), ${ }^{3,5}$ an atomic force microscope (AFM) ${ }^{7}$ or the lateral friction forces between the tip and the sample (shear forces). ${ }^{10,11}$ In all these cases, the tip follows more or less the topography of the sample, and $f(x, y)$ is the convolution of the sample profile by a function which describes the probe geometry.

A comparative study of modes (1) and (2) was presented in Ref. 12. It was shown that the two modes are equivalent, in the sense that a constant-intensity image $z=h(x, y)$ and the constant-height image $\mathscr{S}\left(x, y, z_{0}\right)$, with $z_{0}=\langle h(x, y)\rangle$, are proportional. The brackets denote the background value of a function of $(x, y)$.

Concerning mode (3), the $z$ motion of the probe, induced by a non-optical distance regulation mechanism, can couple to the purely optical information of the image., ${ }^{43}$ This creates an artifact that may lead to a wrong interpretation of the images. It was demonstrated experimentally in Ref. 4 that the SNOM image may contain two different contributions: a purely optical one and one reproducing the motion of the tip. Recently, a systematic experimental study of this artifact was presented, ${ }^{13}$ and showed that many experimental images previously reported might be dominated by non-optical contrast mechanisms. The origin of the artifact was discussed with a simple approach which we summarize here. ${ }^{13,14}$

In the constant-distance mode, we shall write the path followed by the tip as $z=z_{0}+\delta f(x, y)$ where $z_{0}=\langle f(x, y)\rangle$. An expansion of the optical signal $\mathscr{S}$ to first order in $\delta f=\sup |\delta f(x, y)|$ leads to:

$$
\begin{aligned}
\mathscr{S}[x, y, z=f(x, y)]= & \left\langle\mathscr{S}\left(z_{0}\right)\right\rangle+\delta \mathscr{S}\left(x, y, z_{0}\right) \\
& +\frac{\partial \mathscr{S}}{\partial z}\left(x, y, z_{0}\right) \delta f(x, y) .
\end{aligned}
$$

The first term in Eq. (1) is the background value of the image, the second one gives a purely optical contrast and the 
third one reflects the coupling between the optical information and the $z$ motion of the tip. Due to this last term, the optical image will depend on the path followed by the tip $\delta f(x, y)$, leading to the presence of the artifact discussed in Ref. 13. Note that this problem does not occur in constantheight mode because $\delta f(x, y)=0$ and the last term in Eq. (1) always vanishes.

As an illustration, let us consider a worst case scenario. Suppose, for example, that the third term dominates the right-hand side in Eq. (1) and that $\partial \mathscr{P} / \partial z$ is a slowly varying function of $(x, y)$. Then, the detected signal $\mathscr{S}$ given by the left-hand side of Eq. (1) is proportional to the motion of the tip $\delta f(x, y)$. The resulting image is mainly an optical readout of the motion of the tip, as that obtained with a conventional AFM. This image does not contain any information on the optical properties of the sample. Moreover, the resolution of such an image does not result from optical mechanisms, but only from the interaction used to control the tip-sample distance.

The presence or not of this artifact, and its relative weight compared to the purely optical information, depend on both the experimental parameters (as the illumination conditions) and on the sample under study. As pointed out in Ref. 13, this makes the interpretation of constant-distance images a very difficult task. A precise study is necessary and constitutes the scope of the present work. Our purpose is to analyze rigorously the origin of the artifact and to identify the cases in which it may dominate the image contrast. The paper is organized as follows: In Sec. II, we consider the case of weak scattering samples, as that often used in SNOM. We use the three-dimensional perturbation theory to study analytically the origin of the artifact and to discuss the influence of both the experimental parameters and the sample. In Sec. III, we illustrate the discussion of Sec. II with exact two-dimensional numerical simulations based on a resolution of a volume integral (Lippmann-Schwinger) equation for the electric field. The samples studied in Sec. III consist of localized particles (dielectric or metallic) deposited on a flat dielectric substrate. In Sec. IV we study what happens when the sample is a very rough extended surface. In this case the scattered field cannot be described with perturbation theory. This discussion gives a complete picture of the scattering mechanism responsible for the presence of the artifact. Sec. V summarizes our conclusions.

\section{PERTURBATIVE MODEL FOR WEAKLY SCATTERING SAMPLES}

In this section we analyze in detail the origin of the artifact presented in the introduction. We define this artifact as the presence in the detected signal of a cross term between the light scattered by the sample and the $z$ motion of the tip. We will describe analytically the properties of the SNOM images in three dimensions, in the following context:

(1) We focus the discussion on a collection-mode configuration in which the sample is illuminated by an extended field either in reflection or in transmission. After interaction with the sample, the near field is detected by a tip (see Fig. 1). This choice is in no way a limitation of the

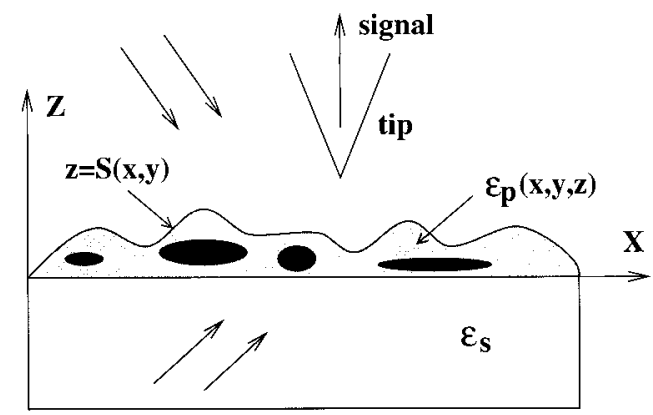

FIG. 1. Section of the three-dimensional geometry used in the perturbation theory.

generality of our study. Reciprocity can be used to extend all the results to the illumination-mode configurations. It has been shown that there exists an equivalent collection-mode setup for any illumination-mode setup. ${ }^{15}$

(2) We assume that the probe is a passive point-like detector. This means that the signal $\mathscr{S}(x, y, z)$ is proportional to the local near-field intensity, defined as the squared modulus of the electric field $|\mathbf{E}(x, y, z)|^{2}$. The passive probe assumption has been studied recently on a rigorous basis. ${ }^{16-18}$ It was also demonstrated that the probe may be passive even if its presence modifies the nearfield distribution around the sample. ${ }^{19,20}$ On this basis, we do not take into account the presence of the tip. In what follows, we shall consider the structure of the nearfield evaluated without the presence of the detecting (or illuminating) tip.

We point out that the coupling efficiency of the probe may depend on $z$. This occurs, for example, under $p$-polarized illumination, when the tip is very close to the sample (a precise study will be reported elsewhere). This $z$ dependence may induce another kind of artifact. This artifact is not studied here because our model does not describe the coupling with the probe.

\section{A. Perturbative expression for the intensity}

We consider a three-dimensional sample with variations in both topography and dielectric constant (Fig. 1). This sample is a layer of profile $z=S(x, y)$ and inhomogeneous isotropic dielectric constant $\epsilon_{p}(x, y, z)$, deposited upon a semi-infinite homogeneous isotropic substrate of dielectric constant $\epsilon_{s}$ (half space $z<0$ ). The upper medium $[z>S(x, y)]$ is assumed to be a vacuum or air. The system is illuminated in transmission or reflection by a monochromatic field of wavelength $\lambda$. This field is either a plane wave (coherent illumination) or a set of uncorrelated plane waves (spatially incoherent illumination). In both cases, the intensity of the incident field depends only on $z$.

Let us write the total near field $\mathbf{E}=\mathbf{E}^{(0)}+\mathbf{E}^{(1)}$ where $\mathbf{E}^{(0)}$ is the field reflected or transmitted by the flat interface $z=0$ and $\mathbf{E}^{(1)}$ is the field scattered by the inhomogeneous layer. In many cases of practical interest in near-field optics (NFO), the object is weakly scattering so that the condition $\left|\mathbf{E}^{(1)}\right|=\eta\left|\mathbf{E}^{(0)}\right|$, with $\eta \ll 1$, is fulfilled. Instances in which 
this condition is not satisfied will be examined in Sec. IV. When the tip is moved along a surface of equation $z=f(x, y)$, the detected intensity to first-order in $\eta$ is:

$$
I[x, y, z=f(x, y)]=I^{(0)}[f(x, y)]+I^{(1)}[x, y, f(x, y)],
$$

where $I^{(0)}=\left|\mathbf{E}^{(0)}\right|^{2}$ and $I^{(1)}=2 \operatorname{Re}\left[\mathbf{E}^{(0)} * \cdot \mathbf{E}^{(1)}\right]$, Re denoting the real part and * the complex conjugate. A first-order expansion of Eq. (2) around $z_{0}=\langle f(x, y)\rangle$ leads to:

$$
\begin{aligned}
I[x, y, z=f(x, y)]= & I^{(0)}\left(z_{0}\right)+\frac{d I^{(0)}}{d z}\left(z_{0}\right) \delta f(x, y) \\
& +I^{(1)}\left(x, y, z_{0}\right) .
\end{aligned}
$$

In order to determine the domain of validity of the preceding equation, we proceed as in Ref. 12. We introduce the length scales $L_{0}$ and $L_{1}$ of $I^{(0)}$ and $I^{(1)}$, respectively, and $\delta f=\sup |\delta f(x, y)|$. Equation (3) is valid if $\delta f \ll L_{1}$ and $\delta f \sim \eta L_{0}$. Note that these conditions involve only the structure of the near field, whatever the physical system which produces this near field. Thus, Eq. (3) applies to a large variety of problems. In the case of a sample with a linear inhomogeneous dielectric permittivity and an arbitrary surface profile, the conditions of validity of Eq. (3) are equivalent to those of first-order perturbation theory in the near field. $^{12,21,22}$

Equation (3) reveals the content of the near-field optical image. The first term is independent on $(x, y)$ and contributes to the background of the image. The two other terms are responsible for the contrast of the image, and two origins for this contrast are clearly identified. The second term is proportional to the path followed by the detecting tip $\delta f(x, y)$. The constant of proportionality depends only on the illumination conditions. Thus this term does not contain any information on the sample, and is only an optical readout of the $z$ motion of the tip. In the best case, i.e., when the tip follows the topography of the sample, this term produces a signal proportional to the topography of the sample, and does not produce any additional information to the shear force, STM, or AFM signal. In contrast, the third term $I^{(1)}\left(x, y, z_{0}\right)$ carries purely optical information on the sample properties (dielectric constant and topography). Its relationship to the topography and the dielectric constant variations of the sample is in general not simple. It can be described with the concepts of impulse response and equivalent surface profile. ${ }^{19}$ This point will be useful in the following discussion.

\section{B. Optical content of the image}

The right-hand side in Eq. (3) shows that the most general image is a superposition of a purely optical signal (third term) and a term proportional to the path followed by the tip (second term). Hence, Eq. (3) gives a rigorous theoretical basis to the experimental observations put forward in Refs. 4 and 13. The relative weight of these two terms determines the optical content of the near-field optical image. If the second term dominates, the use of NFO does not add any information to the AFM, STM, or shear force images. NFO is of interest only in the situations in which the contrast is dominated by the third term in Eq. (3).
Proceeding as in Ref. 13, we introduce the following parameter:

$$
\Gamma=\frac{1}{I^{(1)}\left(x, y, z_{0}\right)} \frac{d I^{(0)}}{d z}\left(z_{0}\right)
$$

which measures the optical content of the image. $\Gamma=0$ corresponds to a purely optical image. A large value of $|\Gamma|$ corresponds to a low optical content of the image, and a domination of the contrast by the artifact. In any relevant NFO image, $|\Gamma|$ should be minimized.

The value of $\Gamma$ depends on both the operating conditions and the properties of the sample under study. This makes the prediction of the presence of this artifact very difficult. We shall address separately the cases corresponding to an illumination in transmission with propagating waves, in transmission with evanescent waves, and in reflection.

\section{Illumination in transmission with propagating waves}

Let us first consider the situation in which the sample is illuminated in transmission from the lower medium (Fig. 1) with a field composed of one monochromatic plane wave (coherent illumination) or a set of uncorrelated plane waves (incoherent illumination), at an angle of incidence smaller than the critical one $\theta_{c}=\arcsin \left[\left(\sqrt{\epsilon_{s}}\right)^{-1}\right]$. The illuminating field $\mathbf{E}^{(0)}$ contains only homogeneous waves, and the illuminating intensity $I^{(0)}$ does not depend on $z\left(d I^{(0)} / d z=0\right)$. Thus, $\Gamma=0$ and no artifact is encountered. ${ }^{23}$ Equation (3) shows that the image in this case is identical to a constantheight image, taken at the height $z=z_{0}$. This is rather surprising because one can have $z_{0}<\sup |S(x, y)|$. In true constant-height mode, one always has $z_{0}>\sup |S(x, y)|$. Thus, we expect that the constant-distance image will look like a constant-height image, but with a better resolution. This will be confirmed by the numerical simulation of Sec. III.

The previous remarks apply to collection-mode SNOM with an illumination in transmission with only homogeneous waves. By reciprocity ${ }^{15}$ it also holds for illumination-mode SNOM in which the light is detected in transmission at angles smaller than the critical one $\theta_{c}$ ("'allowed light" in Ref. 24).

\section{Illumination in transmission with evanescent waves}

The situation in which some or all the plane waves of the incident field have an angle of incidence greater than the critical one is different. The corresponding zero-order transmitted waves are evanescent. Thus, $\mathbf{E}^{(0)}$ contains inhomogeneous waves creating a $z$ dependence in $I^{(0)}$. In the case of a single plane wave, the transmitted zero-order field is of the form $\mathbf{E}_{0} \exp \left(i \mathbf{k}_{\|}^{\text {inc }} \cdot \mathbf{r}_{\|}+i \gamma^{\text {inc }} z\right)$, with $\gamma^{\text {inc }}=\left(k_{0}^{2}-\mathbf{k}_{\|}^{\text {inc } 2}\right)^{1 / 2}$, and $k_{0}=\omega / c$. We have used the notation $\mathbf{r}_{\|}=(x, y)$. An incidence in total internal reflection corresponds to $\left|\mathbf{k}_{\|}^{\text {inc }}\right|>k_{0}$. Thus $\gamma^{\text {inc }}$ is imaginary, with the determination $\operatorname{Im}\left(\gamma^{\text {inc }}\right)>0$, Im denoting the imaginary part. It follows that:

$$
\Gamma=\frac{-2 \operatorname{Im}\left(\gamma^{\text {inc }}\right)}{I^{(1)}\left(x, y, z_{0}\right)}\left|\mathbf{E}_{0}\right|^{2} \exp \left[-2 \operatorname{Im}\left(\gamma^{\text {inc }}\right) z_{0}\right] .
$$


It is worth noting that the numerator of $\Gamma$ (i.e., $d I^{(0)} / d z$ ) is negative in this case. Hence the artifact appears as a superposition to the purely optical image of a signal proportional to the path followed by the tip in inverse contrast. This point will be illustrated by numerical simulation in Sec. III.

The present discussion applies to collection-mode techniques in which the sample is illuminated in transmission with part of (or all) the light being totally internally reflected, as in PSTM/STOM. By reciprocity, ${ }^{15}$ it also applies to the illumination-mode configurations in which the light is detected in transmission at angles greater than the critical one ("forbidden light"' in Ref. 24). The presence of the artifact in "forbidden light" images in illumination mode, and the fact that the non-optical signal represents the path followed by the tip in contrast reversal was demonstrated experimentally in Ref. 13. Our model explains this observation.

\section{Illumination in reflection}

Let us consider now an illumination in reflection from the upper medium (Fig. 1). The illuminating intensity $I^{(0)}$ is an interference pattern between the incident field and the field reflected by the flat interface. Therefore $I^{(0)}$ exhibits a $z$ modulation which may lead to an artifact. This modulation will depend on the value of the reflection factor at the interface $z=0$. Keeping the same notations, in the case of a single incident plane wave, one obtains:

$$
\Gamma=\frac{-4 \gamma^{\mathrm{inc}}}{I^{(1)}\left(x, y, z_{0}\right)} \operatorname{Im}\left[\mathbf{E}_{0}^{*} \cdot \stackrel{\leftrightarrow}{\mathbf{r}} \mathbf{E}_{0} \exp \left(2 i \gamma^{\mathrm{inc}} z_{0}\right)\right]
$$

where $\overleftrightarrow{\mathbf{r}}$ is a matrix of Fresnel reflection factors at the interface $z=0$. Here $\gamma^{\text {inc }}$ is real. Equation (6) shows that the artifact may become important for large values of the reflection factors. Moreover, the sign of the numerator of $\Gamma$ depends on the value of those factors, and may change from one sample to another. Thus the artifact may appear as a signal proportional to the path followed by the tip either in real or in inverse contrast.

The present discussion applies to collection-mode techniques in reflection. By reciprocity it also applies to the illumination-mode techniques in reflection. ${ }^{15}$ In both cases, the artifact will be important if the substrate has a high reflectivity. We will illustrate this point in Sec. III.

\section{Influence of the sample}

The parameter $\Gamma$ defined in Eq. (4) depends on the value of the purely optical contribution $I^{(1)}$. The aim of this section is to analyze the dependence of $I^{(1)}$ on the sample properties.

$I^{(1)}$ is a complicated function of both the experimental conditions and the sample properties. The analysis through perturbation theory leads to the following expression: ${ }^{19}$

$$
I^{(1)}\left(\mathbf{r}_{\|}, z_{0}\right)=\int H\left(\mathbf{r}_{\|}-\mathbf{r}_{\|}^{\prime}, \mathbf{k}_{\|}^{\text {inc }}, \epsilon_{s}, z_{0}\right) S_{\mathrm{eq}}\left(\mathbf{r}_{\|}^{\prime}\right) d \mathbf{r}_{\|}^{\prime} .
$$

$S_{\text {eq }}$ is an equivalent surface profile connecting the topography and the dielectric constant variations of the sample:

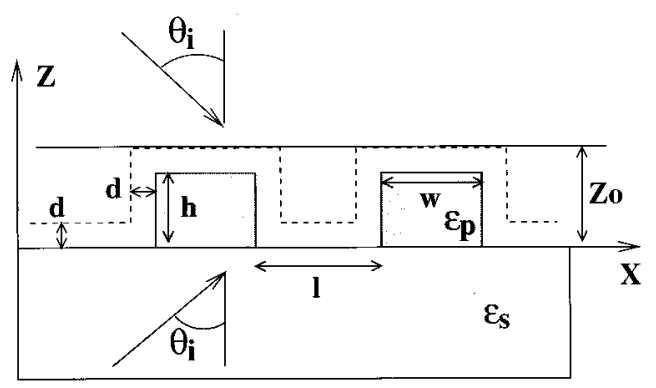

FIG. 2. Geometry of the two-dimensional sample used in the exact numerical calculations. Solid line: path followed in the constant-height calculations. Dashed line: path followed in the constant-distance calculations.

$$
S_{\mathrm{eq}}\left(\mathbf{r}_{\|}\right)=\frac{1}{\epsilon_{s}-1} \int_{0}^{S\left(\mathbf{r}_{\|}\right)}\left[\epsilon_{p}\left(\mathbf{r}_{\|}, z\right)-1\right] d z .
$$

Note that in the case of a homogeneous sample $\left(\epsilon_{p}=\epsilon_{s}\right)$, $S_{\text {eq }}\left(\mathbf{r}_{\|}\right)$reduces to the true topographic profile $S\left(\mathbf{r}_{\|}\right)$. $H$ is an impulse response and is independent on the sample. Its existence is not postulated but comes out from the analysis through first-order perturbation theory. $H$ is known analytically in Fourier space, its expression being given in Ref. 19. $H$ depends on the illumination conditions (polarization, direction of incidence, coherence), on the dielectric constant of the substrate $\epsilon_{s}$ and on the detection distance $z_{0}$. Therefore, $H$ contains the dependence of $I^{(1)}$ on the experimental conditions, while $S_{\text {eq }}$ contains the properties of the sample. These concepts of impulse response and equivalent surface profile describe all the scattering process by weakly scattering samples and are very useful in the description of NFO imaging. ${ }^{19}$

Equation (7) shows that the value of $I^{(1)}$ depends on the relative variations of the functions $H\left(\mathbf{r}_{\|}\right)$and $S_{\mathrm{eq}}\left(\mathbf{r}_{\|}\right)$. Any situation may be encountered. At fixed experimental conditions (both $H$ and $d I^{(0)} / d z$ fixed), a sample may create a strong scattered intensity $I^{(1)}$, thus a small $|\Gamma|$, and another sample a low scattered intensity $I^{(1)}$ and a large $|\Gamma|$. The same problem arises for a given sample by varying the illumination conditions. The conclusion is that it is not possible to give a universal rule governing the presence of the artifact in the image. Nevertheless, it is possible to study a typical sample in order to gain insight. This will be done in the next section.

\section{NUMERICAL RESULTS}

In this section we study the near-field scattered by one or two particles deposited on a flat semi-infinite substrate. The scattering geometry is depicted in Fig. 2. This system is illuminated in transmission or in reflection by a monochromatic plane wave of wavelength $\lambda=633 \mathrm{~nm}$, with an angle of incidence $\theta_{i}$. We provide exact numerical calculations of the total near-field intensity either at a constant height or at a constant distance from the sample. 


\section{A. Numerical method}

The numerical scheme is based on a volume integral formulation of the electric field derived from Maxwell equations. At any point $\mathbf{r}=(x, y, z)$, the electric field is given by: ${ }^{25}$

$$
\mathbf{E}(\mathbf{r})=\mathbf{E}^{(0)}(\mathbf{r})+k_{0}^{2} \int_{\Omega}\left[\epsilon_{p}\left(\mathbf{r}^{\prime}\right)-1\right] \overleftrightarrow{\mathbf{G}}\left(\mathbf{r}, \mathbf{r}^{\prime}\right) \mathbf{E}\left(\mathbf{r}^{\prime}\right) d \mathbf{r}^{\prime},
$$

where $\overleftrightarrow{\mathbf{G}}$ is the Green dyadic of the reference system consisting of a flat interface at $z=0$ separating the substrate (half space $z<0$, dielectric constant $\boldsymbol{\epsilon}_{s}$ ) from vacuum (half space $z>0)$. $\mathbf{E}^{(0)}$ is the field in the reference system and the integral gives an exact expression of the scattered field. It is restricted to the domain $\Omega$ occupied by the particles, having a position-dependent dielectric constant $\epsilon_{p}\left(\mathbf{r}^{\prime}\right)$. In general Eq. (9) can only be solved numerically, ${ }^{26}$ and several schemes have been proposed, in two-dimensional ${ }^{27}$ and three-dimensional geometries. ${ }^{28}$ Here we will consider a two-dimensional geometry, for both $s$ - and $p$-polarized light, and solve Eq. (9) by a moment method. ${ }^{25}$ In this method the volume $\Omega$ is discretized in a mesh of rectangular cells of dimensions $d x$ and $d y$. The field and the dielectric constant are assumed to be constant in each cell. Equation (9) is transformed into a linear system involving the integral of the Green dyadic over each cell. Note that this integration regularized the Green dyadic, which possesses a non-integrable singularity at the origin in $p$ polarization. ${ }^{27}$ In all the calculations presented here, the size of the cells is $d x=d y=0.005 \lambda$.

\section{B. Images of one localized particle with different scattering potentials}

We first consider the sample in Fig. 2 with one single particle. Its width is $w=0.1 \lambda$, its height $h=0.015 \lambda$ and its dielectric constant $\epsilon_{p}$, assumed homogeneous, is a variable parameter. We compare the near-field intensity calculated along a line at a constant height (solid line in Fig. 2) and the intensity calculated at a constant distance from the surface profile (dashed line in Fig. 2). Our goal is to check the presence of the artifact due to the $z$ motion of the tip in the constant-distance mode, in light of the discussion of Sec. II.

\section{Illumination in transmission}

The results in the case of an illumination in transmission at $\theta_{i}=0^{\circ}$ are displayed in Fig. 3 ( $s$ polarization) and Fig. 4 ( $p$ polarization). We have used two different particles having the same dimensions but a different dielectric constant: (a) $\epsilon_{p}=2.25$ (glass), (b) $\epsilon_{p}=-9+i$ (gold). Varying $\epsilon_{p}$ is a way to vary the scattering potential of the sample, and thus the level of the scattered intensity $I^{(1)}$ [see Eqs. (7)-(8)]. The substrate is glass $\left(\epsilon_{s}=2.25\right)$. The solid curves correspond to constant-height calculations along the solid line in Fig. 2 with $z_{0}=0.0225 \lambda(14 \mathrm{~nm})$. The dashed curves correspond to constant-distance calculations along the dashed line in Fig. 2 with $d=0.0075 \lambda(5 \mathrm{~nm})$. The location of the particle is indicated at the bottom of the figures.

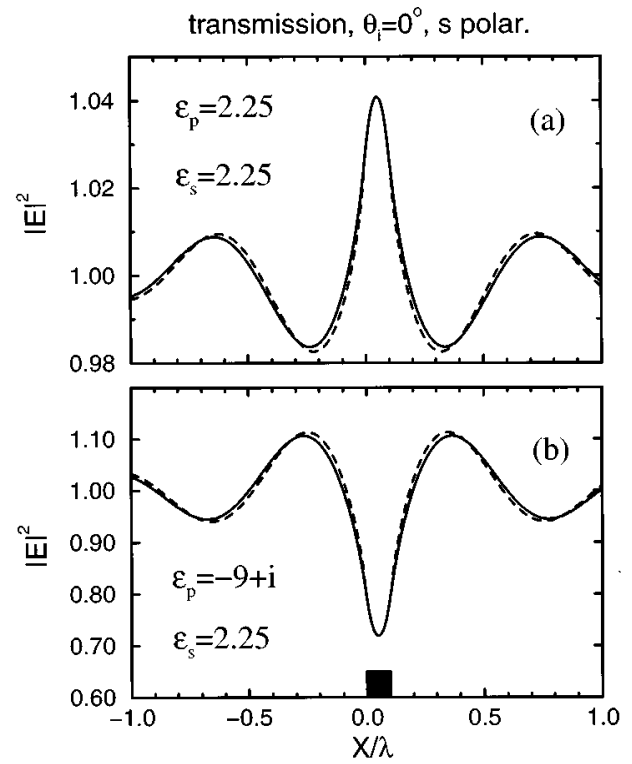

FIG. 3. Near-field intensity above the sample in Fig. 2 with only one surface particle. $s$ polarization; illumination in transmission, $\theta_{i}=0^{\circ}$; particle size: $w=0.1 \lambda, \quad h=0.015 \lambda$. Solid line: constant-height calculations with $z_{0}=0.0225 \lambda$. Dashed line: constant-distance calculations with $d=0.0075 \lambda$. Two values of the particle dielectric constant $\epsilon_{p}$ are used. The dielectric constant of the substrate $\epsilon_{s}=2.25$ is fixed.

In both polarizations and for the two particles, the constant-height and constant-distance curves are similar. This confirms the discussion of Sec. II. At normal incidence in transmission, $d I^{(0)} / d z$ vanishes. Thus, $\Gamma=0$ and the constant-distance image is purely optical. No $z$-motion artifact is to be expected and the constant-height and constantdistance images are similar. Yet the constant-distant curves exhibit a slightly better resolution than the constant-height curves. This is seen in Figs. 3 and 4 where the dashed curves exhibit faster variations than the solid curves. As discussed

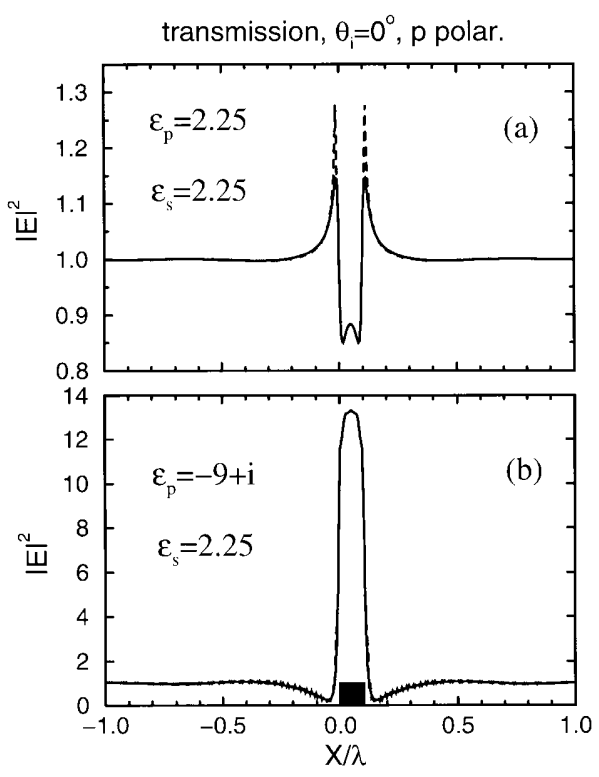

FIG. 4. Same as Fig. 3 for $p$ polarization. 


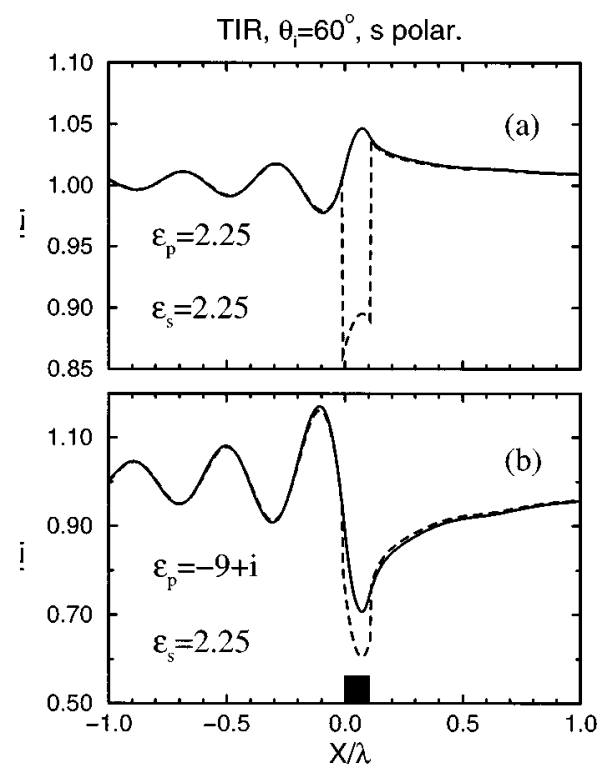

FIG. 5. Same as Fig. 3 with an illumination in total internal reflection $\left(\theta_{i}=60^{\circ}\right)$.

in Sec. II, the constant-distant curve is a constant-height curve taken at the height $\langle f(x, y)\rangle$ which is smaller than the height $z_{0}$ of the constant-height curve. This explains the better resolution.

It is also worth noting the very high level of signal in the case of a metallic particle in $p$ polarization [Fig. 4 (b)]. This is due to an enhancement of the field inside the particle, which creates a very strong scattered field. Even in this case there is no appreciable difference between the two kinds of images.

\section{Illumination in total internal reflection}

We show in Figs. 5 and 6 the same calculations as in Figs. 3 and 4, but for an incidence in total internal reflection $\left(\theta_{i}=60^{\circ}\right)$. As seen in Sec. II, $\Gamma$ does not vanish in this case, its value being given in Eq. (5). Moreover, $d I^{(0)} / d z$ is negative, and one expects a contribution in the intensity of a term proportional to the path followed by the tip in inverse contrast [second term in Eq. (3)]. This is clearly seen in Figs. 5(a) and 6(a). Here the constant-height (solid line) and constant-distance (dashed line) curves look different. A superposition of a purely optical signal (looking like a constant-height signal) and a signal proportional to the path followed by the tip in contrast reversal (artifact) can be easily identified in the constant-distance curves. The scattering potential of the particle being small $\left(\epsilon_{p}=2.25\right)$, the purely optical contribution $I^{(1)}$ does not dominate the contrast of these curves. Thus they are strongly dominated by the $z$-motion artifact.

When the scattering potential increases [Figs. 5(b) and $6(\mathrm{~b})]$, the contribution of the optical term $I^{(1)}$ increases. For a gold particle [Figs. 5(b) and 6(b)], the purely optical contribution is so important that the presence of the artifact is strongly attenuated. This means that the contrast is now dominated by the third term in Eq. (3). The constant-height and constant-distance curves are practically identical. This

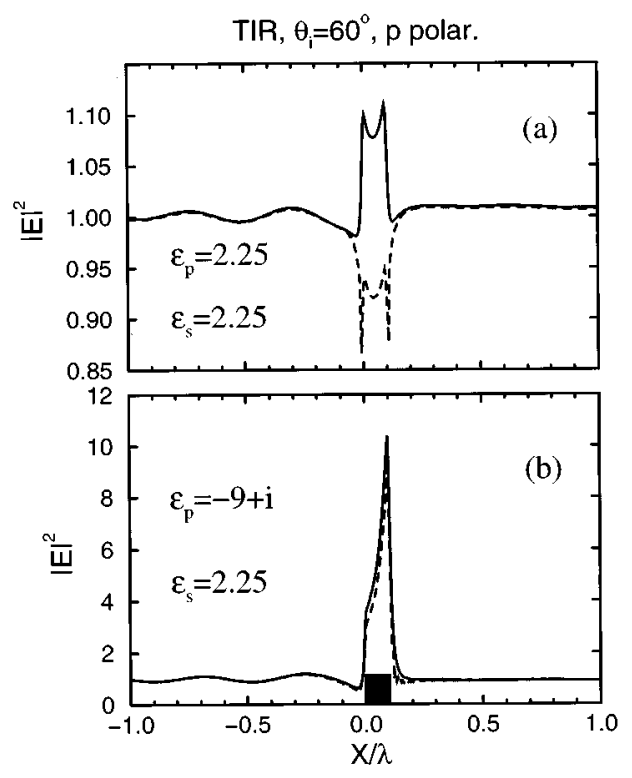

FIG. 6. Same as Fig. 5 for $p$ polarization.

effect is particularly striking in $p$ polarization [Fig. 6(b)] where the metallic particle creates a very strong scattered field. In this case the presence of the artifact is no longer visible.

\section{Illumination in reflection}

We now consider an illumination in reflection from the upper medium (see Fig. 2). We have shown in Sec. II that $\Gamma$ does not vanish in this case. Its expression, given in Eq. (6), shows that its strength should increase with the reflectivity of the substrate. We thus present the calculations for only one kind of particle $\left(\epsilon_{p}=2.25\right)$, but for two values of $\epsilon_{s}:$ (a) $\epsilon_{s}=2.25$ and (b) $\epsilon_{s}=16$. The result is displayed in Fig. 7 ( $s$ polarization). With the values of $\epsilon_{s}$ used here, the Fresnel reflection factors appearing in the matrix $\overleftrightarrow{\mathbf{r}}$ in Eq. (6) are negative. Thus the numerator of $\Gamma\left(d I^{(0)} / d z\right)$ is positive and the $z$-motion artifact should appear as a superposition to the purely optical image of a signal proportional to the path followed by the tip (no contrast reversal). In Fig. 7(a) (low reflective substrate), the constant-height (solid line), and constant-distance (dashed line) curves are only slightly different. The contribution of the artifact in the constantdistance curve is lower than that of the purely optical term. Conversely, in Fig. 7(b) (high reflective substrate), the artifact appears clearly through the addition in the constantdistance intensity of a signal proportional to the path followed by the tip. This calculation confirms that in reflection the artifact is more important when the substrate has a high reflectivity.

Although we do not display the results for the sake of brevity, we have observed the same effect in $p$ polarization. Moreover, increasing the scattering potential of the particle increases the contribution of the optical term in the detected signal. As in the case of TIR examined previously, this reduces the weight of the artifact in the image. 


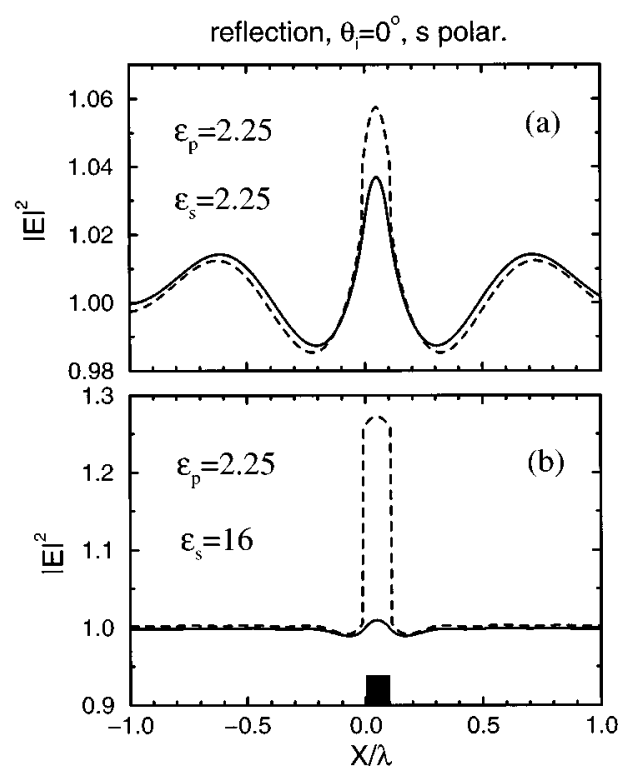

FIG. 7. Near-field intensity above the sample in Fig. 2 with only one surface particle; $s$ polarization. Illumination in reflection from the upper medium, $\theta_{i}=0^{\circ}$. The particle dielectric constant $\epsilon_{p}=2.25$ is fixed. Two values of the substrate dielectric constant $\epsilon_{s}$ are used. Other parameters as in Fig. 3.

\section{Artifact and resolution}

In the previous Section we have shown how the purely optical information of the image could be hidden by the artifact induced by the $z$ motion of the probe. We shall now discuss the resolution issue.

It was pointed out in Ref. 13 that the artifact may lead to a wrong interpretation of the purely optical resolution. We illustrate this important point with exact numerical calculations of the field scattered by two particles $\left(\epsilon_{p}=2.25\right)$ deposited on a flat glass substrate $\left(\epsilon_{s}=2.25\right)$ and separated by a distance $l=0.075 \lambda(47 \mathrm{~nm})$. The geometry is displayed in Fig. 2. The illumination is done in transmission.

Figures 8(a) and 8(b) show the constant-height (solid line) and constant-distance (dashed line) curves for $\theta_{i}=0^{\circ}$ and $\theta_{i}=60^{\circ}$, respectively. The wave is $s$ polarized. In Fig. 8 (a) the presence of the artifact is not visible. As shown previously (see Fig. 3), at normal incidence the constantheight and constant-distance curves are almost identical. The constant-distance curve exhibits a slightly better resolution, as seen in Fig. 8(a). In contrast, in total internal reflection [Fig. 8(b)], the two images are clearly different. In the constant-height curve, the intensity distribution does not reproduce the surface profile. Even the presence of the two particles is not clear in this (purely optical) image. In contrast, the constant-distance curve exhibits strong variations at the precise location of the particles, with an extremely high resolution. But, these strong variations of the signal have their origin in the second term in Eq. (3), which is responsible for the artifact. In fact, a signal proportional to the path followed by the tip in inverse contrast can be easily recognized in the intensity at constant distance. This is the signature of the $z$-motion artifact with an illumination in total internal reflection in collection-mode SNOM (or by reciproc-

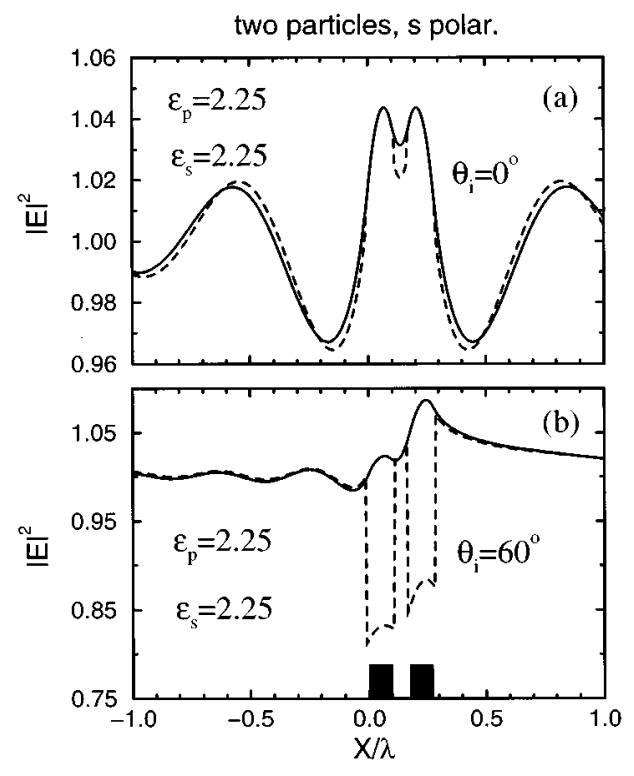

FIG. 8. Near-field intensity above the sample in Fig. 2 with two particles separated by a distance $l=0.075 \lambda$. The dielectric constants $\epsilon_{s}$ and $\epsilon_{p}$ are fixed. (a): $\theta_{i}=0^{\circ} ;(\mathrm{b}): \theta_{i}=60^{\circ}$. Other parameters as in Fig. 3.

ity with a "forbidden light" detection in illumination-mode SNOM). In conclusion, the resolution in the constantdistance image does not have its origin in an optical interaction with the sample, but in an optical readout of the $z$ motion of the tip.

The same behavior is seen in Fig. 9 which is identical to Fig. 8 but for $p$ polarization. Note that the separation between the two particles is clearly resolved in Fig. 9, even in the constant-height images. This is consistent with previous studies that showed that on dielectric substrates the light localization around the object was better in $p$ than in $s$

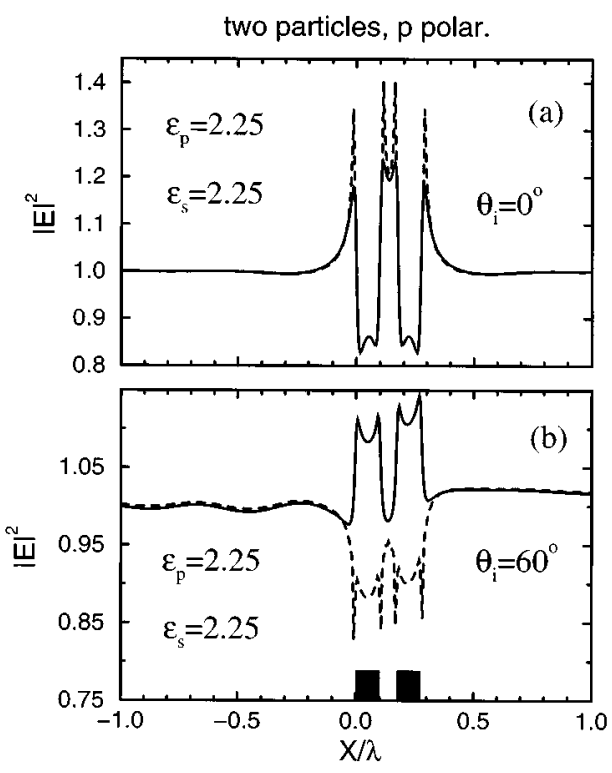

FIG. 9. Same as Fig. 8 for $p$ polarization. 
polarization. ${ }^{21,28}$ Note that this polarization effect may be different with metallic substrates supporting polaritons. ${ }^{29}$ In Fig. 9(b), due to the presence of the artifact, the constantdistance image appears in contrast reversal. But once again, this contrast reversal is a pure effect of the artifact, and does not reveal any particular optical property of the sample.

\section{CASE OF A STRONGLY SCATTERING EXTENDED SURFACE}

The analysis in Sec. II, based on perturbation theory, shows that the origin of the $z$-motion artifact is the $z$ variation of the illuminating intensity $d I^{(0)} / d z$. This term competes with the first-order scattered intensity $I^{(1)}$ to produce the contrast of the image. In the perturbative analysis of Sec. II, the $z$ variation of $I^{(1)}$ was neglected because it was a second-order contribution. We have seen that this model predicts the behavior of the images of small localized particles, dielectric or metallic, as that studied numerically in Sec. III. This study is relevant for NFO applications.

Nevertheless, in order to get a complete picture of the scattering process responsible for the $z$-motion artifact, in this section we shall analyze the behavior of a strongly scattering extended non-flat surface. Note that, with constantdistance regulation, it is possible to study such highly corrugated samples with NFO microscopes. ${ }^{30}$ In this case the scattered intensity is no longer weak compared to the illuminating intensity, and it may even dominate the total intensity. Hence, presently, the perturbative development used in Sec. II is not meaningful. This is a well-known fact in scattering from rough surfaces: when the roughness increases, the amount of energy in the specular direction (i.e., $I^{(0)}$ ) decreases, the energy being transferred to the scattered part of the field. In this case we may expect that the $z$ variation of the scattered intensity may induce a new $z$-motion artifact.

In order to check this hypothesis, we have calculated the near-field scattered by a one-dimensional grating of profile $z=S(x)$ with $S(x)=h \cos (2 \pi x / p)$. The profile is represented on the top in Fig. 10. The upper medium is a vacuum, while the lower medium is assumed to be glass $\left(\epsilon_{s}=2.25\right)$. The period is $p=0.5 \lambda$. The sample is illuminated in transmission from the lower medium with a monochromatic plane wave $(\lambda=633 \mathrm{~nm})$ at normal incidence.

We show in Fig. 10(a) the calculations when $h=0.01 \lambda$, in $s$ polarization. The solid curve corresponds to a constant-height calculation, the dashed line to a constantdistance calculation. As expected, with this smooth grating, the conclusion of Secs. II and III remains valid. At normal incidence in transmission, the two curves are almost identical and no $z$-motion artifact may be detected in the constantdistance curve. It is so because the small grating height makes it a smooth scattering sample, for which the first-order perturbation theory is valid. Thus, Eq. (3) correctly describes the behavior of the near-field intensity and the $z$ variation of the scattered intensity is a negligible second-order correction.

We show in Fig. 10(b) the same calculation with $h=0.15 \lambda$. The result is completely different. Even at normal incidence in transmission, the two curves are not identical. A $z$-motion artifact appears, but its origin is not the $z$ variation

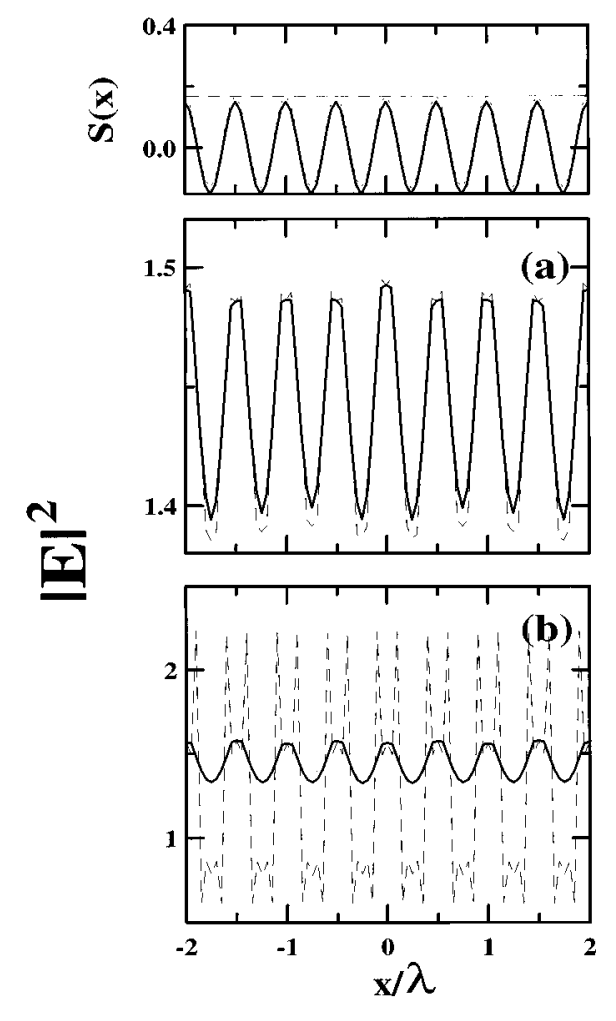

FIG. 10. Near-field intensity above a homogeneous surface of profile $S(x)=h \cos (2 \pi x / p)$, with $p=0.5 \lambda$. Dielectric constant of the surface $\epsilon_{s}=2.25$. Solid line: constant-height calculation. Dashed line: constantdistance calculation; $s$ polarization: $\theta_{i}=0^{\circ}$. (a): $h=0.01 \lambda ;(\mathrm{b}): h=0.15 \lambda$.

of $I^{(0)}$. In fact, the mechanism is the following. The total electric field can be written $\mathbf{E}=\mathbf{E}^{(0)}+\mathbf{E}_{s}$. In this decomposition $\mathbf{E}_{s}$ denotes the scattered field. The total intensity is now:

$$
I(\mathbf{r})=I^{(0)}(\mathbf{r})+2 \operatorname{Re}\left[\mathbf{E}^{(0) *}(\mathbf{r}) . \mathbf{E}_{s}(\mathbf{r})\right]+I_{s}(\mathbf{r}),
$$

where $I_{s}=\left|\mathbf{E}_{s}\right|^{2}$. The high-roughness grating creates a strong scattered field $\mathbf{E}_{s}$, and the last two terms in Eq. (10) contribute to the scattered intensity. The last one is not negligible, and may even dominate. Moreover, because $\mathbf{E}_{s}$ contains both propagating and evanescent waves, the scattered intensity depends strongly on $z$, and induces the $z$-motion artifact proportional to the $z$ derivative of the scattered intensity. Calculations of the total intensity versus $z$ close to the surface (not displayed here) show that the derivative of the intensity is four times greater in the case in Fig. 10(b) than that in Fig. 10(a). This explains the visibility of a $z$-motion artifact in Fig. 10(b).

Also, not shown for the sake of brevity, the same results have been obtained in $p$ polarization.

\section{CONCLUSION}

For weakly scattering samples, the scattering process does not induce any artifact in collection-mode SNOM when the sample is illuminated in transmission with propagating waves. This also holds for illumination-mode SNOM with a detection in transmission at angles smaller than the critical one. In collection-mode SNOM with some of the waves be- 
ing totally internally reflected (like in PSTM/STOM), the artifact may become important. This is also true in illumination-mode SNOM with detection in transmission at angles greater than the critical one. In reflection SNOM, the artifact may also be encountered. It is important when the substrate has a high reflectivity. We have also shown that the purely optical contribution in the image increases with the scattering potential of the sample.

For strongly scattering extended surfaces, the scattering process induces an artifact even in transmission with illuminating (or detected) waves propagating with an angle of incidence smaller than the critical one.

The artifact we have studied is induced by the scattering process by the sample. As pointed out in the introduction, the probe coupling efficiency may depend on $z$ and induces another kind of artifact, which was not taken into account here. Situations in which we did not find any artifact (of the first kind) may be affected in experiments by the probe-coupling artifact.

A safe way to avoid the artifact studied here would be to perform constant-height (or constant-intensity) images. Nevertheless, the constant-distance mode allows to perform a scan at a mean distance $z_{0}$ smaller than the maximum height of the surface profile. Thus this mode provides the best potential optical resolution. We would like to address the problem of extracting the purely optical information from a constant-distance image exhibiting a $z$-motion artifact. In an experiment, one measures both the "optical signal" described by the left-hand side in Eq. (3) and the $z$ motion $\delta f(x, y)$ of the tip. The illuminating intensity $I^{(0)}$ and its derivative $d I^{(0)} / d z$ are known quantities that can be evaluated from the experimental parameters. Therefore solving for Eq. (3) gives a general procedure to extract the purely optical information $I^{(1)}$ from the experimental signal. Such a procedure has been used experimentally in Ref. 31 on a collectionmode image obtained with an illumination in total internal reflection. A signal proportional to the path followed by the tip in inverse contrast was extracted from the image, leading to a purely optical signal. The main problem in this procedure is that the level of the purely optical signal in the input image has to be above the level of noise. Our study allows to find conditions in which the purely optical information is enhanced.

\section{ACKNOWLEDGMENTS}

The authors thank D.W. Pohl for helpful discussions. This research has been supported by the Comision Interministerial de Ciencia y Tecnologia under Grant PB-95-0061A and by the EC. R.C. acknowledges financial support from the ESPRIT Program of the European Union. A.M. acknowledges a scholarship from Comunidad de Madrid.
${ }^{1}$ Near-Field Optics, edited by D. W. Pohl and D. Courjon, NATO ASI Series E (Kluwer, Dordrecht, 1993), Vol. 242.

${ }^{2}$ Optics at the Nanometer Scale, edited by M. Nieto-Vesperinas and N. García, NATO ASI Series E (Kluwer, Dordrecht, 1996), Vol. 319.

${ }^{3}$ U. Dürig, D. W. Pohl, and F. Rohner, J. Appl. Phys. 59, 3318 (1986).

${ }^{4}$ E. Betzig, in Near-Field Optics, edited by D. W. Pohl and D. Courjon, NATO ASI Series E (Kluwer, Dordrecht, 1996), Vol. 242.

${ }^{5}$ J. Koglin, U.Ch. Fischer, and H. Fuchs, in Optics at the Nanometer Scale, edited by M. Nieto-Vesperinas and N. Garciá, NATO ASI Series E (Kluwer, Dordrecht, 1996), Vol. 319, p. 247.

${ }^{6}$ E. Betzig, M. Isaacson, and A. Lewis, Appl. Phys. Lett. 51, 2088 (1987).

${ }^{7}$ N. F. van Hulst, M. H. P. Moers, and B. Bölger, J. Microsc. 171, 95 (1993).

${ }^{8}$ F. Zenhausern, M. P. O’Boyle, and H. K. Wickramasinghe, Appl. Phys. Lett. 65, 1623 (1994); S. Kawata and Y. Inouye, Ultramicroscopy 57, 313 (1995); R. Bachelot, P. Gleyzes, and A. C. Boccara, Opt. Lett. 20, 1924 (1995).

${ }^{9}$ R. C. Reddick, R. J. Warmack, and T. L. Ferrel, Phys. Rev. B 39, 767 (1989); D Courjon, K. Sarayeddine, and M. Spajer, Opt. Commun. 71, 23 (1989); F. de Fornel, J. P. Goudonnet, L. Salomon, and E. Lesniewska, Proc. SPIE 1139, 77 (1989).

${ }^{10}$ E. Betzig, P. L. Finn, and S. J. Weiner, Appl. Phys. Lett. 60, 2484 (1992).

${ }^{11}$ R. Toledo-Crow, P. C. Yang, and M. Vaez-Iravani, Appl. Phys. Lett. 60, 2957 (1992).

${ }^{12}$ R. Carminati and J.-J. Greffet, Opt. Lett. 21, 1208 (1996).

${ }^{13}$ B. Hecht, H. Bielefeldt, Y. Inouye, D. W. Pohl, and L. Novotny, J. Appl. Phys. 81, 2492 (1997).

${ }^{14}$ R. Carminati, Ph.D thesis, Ecole Centrale Paris, France, 1996.

${ }^{15}$ E. R. Méndez, J.-J. Greffet, and R. Carminati, Opt. Commun. (in press).

${ }^{16}$ R. Carminati and J.-J. Greffet, Opt. Commun. 116, 316 (1995).

${ }^{17}$ A. Madrazo and M. Nieto-Vesperinas, J. Opt. Soc. Am. A 13, 785 (1996); ibid. 14, 618 (1997).

${ }^{18}$ J. C. Weeber, F. de Fornel, and J. P. Goudonnet, Opt. Commun. 126, 285 (1996).

${ }^{19}$ J.-J. Greffet and R. Carminati, in Optics at the Nanometer Scale, edited by M. Nieto-Vesperinas and N. Garciá, NATO ASI Series E (Kluwer, Dordrecht, 1996), Vol. 319, p. 1.

${ }^{20}$ A. Madrazo and M. Nieto-Vesperinas, J. Opt. Soc. Am. A (in press).

${ }^{21}$ R. Carminati and J.-J. Greffet, J. Opt. Soc. Am. A 12, 2716 (1995).

${ }^{22}$ D. Barchiesi, C. Girard, O. J. F. Martin, D. Van Labeke, and D. Courjon, Phys. Rev. E 54, 4285 (1996).

${ }^{23}$ The $z$ variation of $I^{(1)}$ could induce an artifact, but this variation is of second order in $\eta$ and is not taken into account in Eq. (3). It induces a visible effect only in the case of a highly scattering extended surface (see Sec. IV).

${ }^{24}$ B. Hecht, H. Heinzelmann, and D. W. Pohl, Ultramicroscopy 57, 228 (1995).

${ }^{25}$ R. Harrington, Field Computation by Moment Methods (IEEE, Piscataway, NJ, 1993).

${ }^{26}$ Approximate analytical expressions of the field can be derived from Eq. (9) by using the Born approximation. This leads to the first-order perturbation theory used in Sec. II. See Ref. 21 for more details.

${ }^{27}$ F. Pincemin, A. Sentenac, and J.-J. Greffet, in Near-Field Optics, edited by D. W. Pohl and D. Courjon, NATO ASI Series E (Kluwer, Dordrecht, 1996), Vol. 242, p. 209; F. Pinceman, A. Sentenac, and J.-J. Greffet, J. Opt. Soc. Am. A 11, 1117 (1994).

${ }^{28}$ O. J. F. Martin, C. Girard, and A. Dereux, Phys. Rev. Lett. 74, 526 (1995).

${ }^{29}$ A. Madrazo and M. Nieto-Vesperinas, Appl. Phys. Lett. 70, 31 (1997).

${ }^{30}$ R. Toledo-Crow, B. Smith, J. Rogers, and M. Vaez-Iravani, Proc. SPIE 2196, 62 (1994).

${ }^{31}$ Y. Martin, F. Zenhausern, and H. K. Wickramasinghe, Appl. Phys. Lett. 68, 2475 (1996). 